\title{
Tailoring bacterial taxa for immune cell modulation
}

\author{
Ofer Margalit ${ }^{1,2}$, Ben Boursi $i^{1,2,3}$ \\ ${ }^{1}$ Department of Oncology, Sheba Medical Center, Ramat Gan, Israel; ${ }^{2}$ Tel-Aviv University, Tel-Aviv, Israel; ${ }^{3}$ Center for Clinical Epidemiology and \\ Biostatistics, Perelman School of Medicine at the University of Pennsylvania, Philadelphia, PA, USA \\ Correspondence to: Ben Boursi, MD. Department of Oncology, Sheba Medical Center, Ramat Gan, Israel; Tel-Aviv University, Tel-Aviv, Israel; \\ Center for Clinical Epidemiology and Biostatistics, Perelman School of Medicine at the University of Pennsylvania, Philadelphia, PA, USA. \\ Email: ben.boursi@sheba.health.gov.il. \\ Comment on: Schluter J, Peled JU, Taylor BP, et al. The gut microbiota is associated with immune cell dynamics in humans. Nature 2020;588:303-7.
}

Submitted Jun 24, 2021. Accepted for publication Jul 28, 2021.

doi: 10.21037/hbsn-21-263

View this article at: https://dx.doi.org/10.21037/hbsn-21-263

The gut microbiota is being increasingly recognized as having a major role in modulating the immune system (1) and response to immunotherapy $(2,3)$. Landmark preclinical studies have shown that gut microbiota transferred via fecal microbiota transplantation (FMT) from humans or mice that have responded to immunotherapy enhanced the efficacy of anti-PD-L1 and anti-CTLA-4 in recipient mice $(2,3)$. Hence, the therapeutic efficacy of immunotherapy depends upon microbiota composition. This conclusion led to the initiation of several clinical trials in which FMT was used to overcome resistance to immunotherapy in human patients. The first two proof-of-concept clinical trials involved metastatic melanoma patients that were converted from immunotherapy-refractory to immunotherapy-responsive using FMT from durable responders to immunotherapy $(4,5)$. Possible direct and indirect mechanisms underlying this response include nonspecific immune activation (6), molecular mimicry (7) and presentation of bacterial peptides by tumor cells (8). Nonspecific immune activation via FMT was previously shown in mouse models to increase myeloid and lymphocyte counts in both the bone marrow and peripheral blood (9-11). Collectively, these data emphasize the need to identify specific bacterial taxa associated with immune cell counts and explore the dynamics of these associations.

In a recently published article in Nature, Schluter et al. investigated the association between gut microbiota and immune cell dynamics in humans (12). The authors selected a favorable setting for evaluating this association by analyzing data from patients undergoing hematopoietic cell transplantation (HCT). This intervention, which both depletes white blood cell (WBC) counts and damages the gut microbiota, established a common baseline for the analysis. The study was comprised of two separate components. In the first part, the authors were seeking to detect an association between microbiota and circulatory WBCs. They used data from a randomized trial of 24 patients with various hematologic malignancies, including myelodysplastic syndrome (MDS), chronic lymphocytic lymphoma (CLL), multiple myeloma (MM), acute lymphocytic leukemia (ALL) and acute myelogenous leukemia (AML) (12). All patients received HCT, with a variability in conditioning intensity as well as in HCT type, i.e., peripheral blood stem cells (PBSC), T cell depleted graft, bone marrow or umbilical cord blood. In this randomized trial, 14 patients received autologous FMT (auto-FMT) and 10 patients served as controls. The authors compared neutrophil, lymphocyte and monocyte counts in peripheral blood between the two treatment arms, and found an association between auto-FMT and increased WBC counts. Based on the positive results of the first part, the authors proceeded to the second part, which included two sequential stages. Stage 1 served as a clinicaland metadata-feature-selection stage using blood and medication data from a large observational cohort of 1,096 patients receiving allo-HCT without available microbiome information. In this stage, the authors identified HCT type, conditioning intensity, medications and clinical features (i.e., age and sex) as associated with immune reconstitution kinetics. In the following stage 2, the authors aimed to define an association between specific bacterial taxa and WBCs dynamics. This stage included 841 patients from 
an independent cohort with available microbiome samples. The authors found an association between Ruminococcus 2 abundance and increased lymphocyte counts, as well as an association between Ruminococcus 2, Faecalibacterium and Akkermansia abundance and increased neutrophil counts. The effect of granulocyte-colony stimulating factor (GSCF) administration in patients with higher levels of these three bacterial taxa was accelerated compared with patients with lower levels, with a 2.4-days difference in reaching immune reconstitution. This study had two methodological caveats. First, only $40 \%$ of stool samples were taken after neutrophil engraftment. Second, only PBSC-recipients were included in the main analysis. In conclusion, Schluter et al. showed for the first time an association between peripheral WBC dynamics and specific bacterial taxa, namely, Ruminococcus 2, Faecalibacterium and Akkermansia. These findings suggest a potential role for microbiota-mediated modulation of the immune system based on the differential effect of each of the specific bacterial taxa on WBC dynamics.

The study by Schluter et al. reconciles two previous studies suggesting Ruminococcus and Faecalibacterium (13) as well as Akkermansia (14) as associated with an improved response to anti-PD-1 immunotherapy. However, their study falls short of recapitulating previous data linking additional bacterial taxa with response to immunotherapy. For example, Bifidobacterium longum was shown to be associated with a response to immunotherapy (15), while Bacteroidales order was associated with a lack of response (13). Possible explanations may stem from the fact that Schluter et al. focused on post-HCT immune reconstitution in the peripheral blood, but did not assess immune function or lymphocyte infiltration of target tissue. Moreover, the study did not include immunotherapy administration to patients. An additional possible confounder may be the use of antibiotics at various timepoints following HCT in all patients included in the main analysis, expected to result in microbiota depletion. This questions the suggested causality between specific bacterial taxa and WBC dynamics.

The study by Schluter et al. opens new avenues for manipulating specific components of the immune system through a corresponding microbiota-driven modulation. Future studies in the field of microbiota and cancer should explore immune cell dynamics within the tumor, characterize specific myeloid and lymphocyte subpopulations affected by FMT, with an emphasis on CD8+ effector $\mathrm{T}$ cells, and look for a possible correlation between WBC dynamics and combinations of several bacterial taxa.

\section{Acknowledgments}

Funding: None.

\section{Footnote}

Provenance and Peer Review: This article was commissioned by the editorial office of Hepatobiliary Surgery and Nutrition. The article did not undergo external peer review.

Conflicts of Interest: Both authors have completed the ICMJE uniform disclosure form (available at https://hbsn. amegroups.com/article/view/10.21037/hbsn-21-263/coif). The authors have no conflicts of interest to declare.

Ethical Statement: The authors are accountable for all aspects of the work in ensuring that questions related to the accuracy or integrity of any part of the work are appropriately investigated and resolved.

Open Access Statement: This is an Open Access article distributed in accordance with the Creative Commons Attribution-NonCommercial-NoDerivs 4.0 International License (CC BY-NC-ND 4.0), which permits the noncommercial replication and distribution of the article with the strict proviso that no changes or edits are made and the original work is properly cited (including links to both the formal publication through the relevant DOI and the license). See: https://creativecommons.org/licenses/by-nc-nd/4.0/.

\section{References}

1. Belkaid Y, Hand TW. Role of the microbiota in immunity and inflammation. Cell 2014;157:121-41.

2. Sivan A, Corrales L, Hubert N, et al. Commensal Bifidobacterium promotes antitumor immunity and facilitates anti-PD-L1 efficacy. Science 2015;350:1084-9.

3. Vétizou M, Pitt JM, Daillère R, et al. Anticancer immunotherapy by CTLA-4 blockade relies on the gut microbiota. Science 2015;350:1079-84.

4. Baruch EN, Youngster I, Ben-Betzalel G, et al. Fecal microbiota transplant promotes response in immunotherapy-refractory melanoma patients. Science 2021;371:602-9.

5. Davar D, Dzutsev AK, McCulloch JA, et al. Fecal microbiota transplant overcomes resistance to anti-PD-1 therapy in melanoma patients. Science 2021;371:595-602.

6. Sepich-Poore GD, Zitvogel L, Straussman R, et al. The microbiome and human cancer. Science 
2021;371:eabc4552.

7. Fluckiger A, Daillère R, Sassi M, et al. Cross-reactivity between tumor MHC class I-restricted antigens and an enterococcal bacteriophage. Science 2020;369:936-42.

8. Kalaora S, Nagler A, Nejman D, et al. Identification of bacteria-derived HLA-bound peptides in melanoma. Nature 2021;592:138-43.

9. Deshmukh HS, Liu Y, Menkiti OR, et al. The microbiota regulates neutrophil homeostasis and host resistance to Escherichia coli K1 sepsis in neonatal mice. Nat Med 2014;20:524-30.

10. Schwab L, Goroncy L, Palaniyandi S, et al. Neutrophil granulocytes recruited upon translocation of intestinal bacteria enhance graft-versus-host disease via tissue damage. Nat Med 2014;20:648-54.

Cite this article as: Margalit O, Boursi B. Tailoring bacterial taxa for immune cell modulation. HepatoBiliary Surg Nutr 2021;10(5):686-688. doi: 10.21037/hbsn-21-263
11. Zhang D, Frenette PS. Cross talk between neutrophils and the microbiota. Blood 2019;133:2168-77.

12. Schluter J, Peled JU, Taylor BP, et al. The gut microbiota is associated with immune cell dynamics in humans.

Nature 2020;588:303-7.

13. Gopalakrishnan V, Spencer CN, Nezi L, et al.

Gut microbiome modulates response to anti-PD-1 immunotherapy in melanoma patients. Science 2018;359:97-103.

14. Routy B, Le Chatelier E, Derosa L, et al. Gut microbiome influences efficacy of PD-1-based immunotherapy against epithelial tumors. Science 2018;359:91-7.

15. Matson V, Fessler J, Bao R, et al. The commensal microbiome is associated with anti-PD-1 efficacy in metastatic melanoma patients. Science 2018;359:104-8. 